\title{
Ground-based observations of diffuse auroral structures in conjunction with Reimei measurements
}

\author{
M. Samara ${ }^{1}$, R. G. Michell ${ }^{1}$, K. Asamura ${ }^{2}$, M. Hirahara ${ }^{3}$, D. L. Hampton ${ }^{4}$, and H. C. Stenbaek-Nielsen ${ }^{4}$ \\ ${ }^{1}$ Southwest Research Institute, San Antonio, TX, USA \\ ${ }^{2}$ Institute of Space and Astronautical Science/Japan Aerospace Exploration Agency, Kanagawa, Japan \\ ${ }^{3}$ Department of Earth and Planetary Science, The University of Tokyo, Tokyo, Japan \\ ${ }^{4}$ The University of Alaska, Fairbanks, Fairbanks, AK, USA
}

Received: 2 October 2009 - Revised: 18 March 2010 - Accepted: 23 March 2010 - Published: 26 March 2010

\begin{abstract}
We present results from ground-based auroral observations coordinated with the Japanese satellite, Reimei, that took place during the winters of 2006, 2007 and 2008 at Poker Flat, Alaska. Comparable temporal and spatial resolution for the optical and in situ particle data, allowed for investigation of small scale and/or rapidly time-varying auroral structures. Four satellite passes through diffuse auroral structures were identified. The structures within the aurora, whether stationary or time-varying (pulsating aurora), were most closely correlated with the highest energy precipitating electrons measured by these detectors ( 8 to $12 \mathrm{keV}$ ). This relation is found to be consistent across all four examples, revealing that the electron precipitation responsible for these diffuse auroral structures is primarily that of the $\geq 8 \mathrm{keV}$ electrons.
\end{abstract}

Keywords. Ionosphere (Auroral ionosphere) - Magnetospheric physics (Energetic particles, precipitating; Magnetosphere-ionosphere interactions)

\section{Introduction}

Ground based auroral observations have typically focused on the discrete structures often associated with activations and substorms, partly due to their brightness and dynamic nature. Recently, however, there has been increased interest in understanding the diffuse auroral emissions (Horne et al., 2003; Dashkevich et al., 2007; Ni et al., 2008; Sergienko et al., 2008). In particular, periodically varying (spatially and temporally) structure within the diffuse aurora can be used to study wave activity (Johnstone et al., 1993; Horne and

Correspondence to: M. Samara

(msamara@swri.edu)
Thorne, 2000; Meredith et al., 2000; Peticolas et al., 2002). The extent, intensity and spectra of the auroral emissions are an indicator of energy input into the ionosphere from the magnetosphere.

The first large-scale survey of horizontal auroral thickness, Maggs and Davis (1968), found that rayed auroral structures tended to be thinner than diffuse ones and that all types of aurora tended to get brighter as they got thinner. Since then there have been numerous studies of these thinner, active, substorm auroral arcs such as Haerendel et al. (1994); Stenbaek-Nielsen et al. (1998); Hallinan et al. (2001). However, a more recent examination of the Maggs and Davis (1968) data set by Stenbaek-Nielsen et al. (1999), revealed that a significant fraction of those observations were taken inside diffuse aurora, showcasing that the context in which auroral research takes place is largely defined by it. In addition, diffuse auroral structures make up the majority of the aurora, and contain significant energy input from the magnetosphere into the polar ionosphere (Meredith et al., 2009).

The phenomenon of black aurora, typically observed within diffuse aurora, has been studied since the 1970s. It is defined as a lack of auroral emissions in a small, well-defined region embedded within a uniform background of diffuse aurora or aurora that is intermediate between diffuse and discrete (Royrvik, 1976). There were several large morphological studies of black aurora in the 1990s, describing its characteristics and features (such as, Schoute-Vanneck et al., 1990; Trondsen and Cogger, 1997; Kimball and Hallinan, 1998). More recent studies, using satellites (Peticolas et al., 2002), and radars (Blixt and Kosch, 2004; Blixt et al., 2005) have linked the generation of black aurora to a magnetospheric origin. Therefore its presence in ground-based auroral images can be used to gain information about in situ processes and assist in interpreting combined in situ data.

Published by Copernicus Publications on behalf of the European Geosciences Union. 
Studies combining in situ measurements and groundbased auroral imaging, provide insight into the acceleration processes associated with various auroral structures. Previous studies have focused on active aurora (Hallinan et al., 2001; Stenbaek-Nielsen et al., 1998), but less on diffuse structures, such as Peticolas et al. (2002). More recently, Sergienko et al. (2008) investigated the fine structure of diffuse aurora. It is this diffuse aurora, with varying amounts of sub-structure, that we seek to understand with the conjunctive events presented in this paper. The changes in luminosity of these small scale features are explored in relation to high resolution in situ particle measurements, identifying the precipitating electron populations responsible.

Diffuse aurora, refers to aurora caused by precipitation from populations of trapped magnetospheric electrons. These are scattered into the loss cone by interactions with waves, primarily chorus and $\mathrm{ECH}$, in the equatorial region of the plasma sheet. The electron spectra associated with diffuse aurora are primarily Maxwellian and do not contain signs of acceleration by parallel electric fields.

\section{Method}

Observational campaigns were conducted from Poker Flat, Alaska recording ground based imager data, which are combined with in situ satellite observations. Poker Flat, at a geodetic location of $65.1 \mathrm{~N}, 212.6 \mathrm{E}(65.3 \mathrm{~N}, 263.2 \mathrm{E}$ Geomagnetic), corresponds to an L-value of 5.7, with local magnetic midnight occurring at approximately 11:20 UT. The optical data presented are extracted from an intensified CCD all-sky imager, with a NTSC video output containing an accurate GPS time stamp. They were recorded to DVD at 30 frames per second. The resolution is approximately 3 pixels per degree, or about 500 to $600 \mathrm{~m}$ per pixel in the vicinity of the zenith. The imager is unfiltered, recording the whitelight auroral emissions that are primarily from the green line $(5577 \AA)$, which in turn dominates the extracted intensities.

The in situ data are from the Japanese Reimei satellite, which is in sun-sunchronous polar orbit at $\sim 650 \mathrm{~km}$ altitude. It contains high resolution, $40 \mathrm{~ms}$ per spectra with 32 energy steps, ion and electron spectrometers covering the range of $12 \mathrm{eV}$ to $12 \mathrm{keV}$ (Asamura et al., 2003). The velocity of Reimei is approximately $9 \mathrm{~km} / \mathrm{s}$, resulting in a spatial resolution of $380 \mathrm{~m}$, which is less than the spatial resolution of the imager, thus not affecting the comparisons.

Four Reimei overpasses are examined, one each from 2006 and 2007, and two from 2008, where diffuse auroral structures were present in the all-sky data. Each overpass is represented by a three part panel of identical format for comparison (Figs. 2, 3, and 4).

To best understand the nature of the auroral features that Reimei encountered, a type of keogram (a) is constructed, using intensity cuts taken along the trajectory of the footpoint of the satellite. Specifically, at each frame, an inten- sity cut is taken along the path of the magnetic footpoint at $100 \mathrm{~km}$ altitude, covering the closest approach to zenith at Poker Flat over a fixed time period of $\sim$ one minute. For example, in Fig. 2a (left panel) the intensity cut is taken along the footpoint position from 12:01:10 to 12:01:50 UTC. When stacked into an image, the intensity at the exact spacecraft footpoint can be read off the diagonal line from lower left to upper right. From this representation one can determine whether the features seen in the particle data come from spatial or temporal variations in auroral intensity. Reimei always passes over Poker Flat in a North-to-South direction, with the result of North reflected at the bottom and South at the top of the keogram.

Panel (c), shows a series of typical Reimei energy spectrograms for electrons and ions, at different pitch angle ranges.

Panel (b) combines information from panels (a) and (c). The black curve shows the optical intensity of the aurora at the footpoint location. It is extracted from the all-sky data, on an arbitrary scale of 30 (dark) to 255 (bright), and matches the location of the line in (a). The coloured lines are the energy flux in different energy ranges, where the blue $(8-12 \mathrm{keV})$, green $(100-1000 \mathrm{eV})$ and red $(10-100 \mathrm{eV})$ lines, are extracted from the Reimei electron data for each energy range noted. The flux of the downgoing electrons $\left(0^{\circ}\right.$ to $\left.30^{\circ}\right)$ is summed within each energy range. These three lines have been scaled for comparison with the optical line. The green and red lines are scaled by a factor of 10 relative to the blue line.

The common $\mathrm{x}$-axis shows the time in UTC. The y-axes show (a) the distance along the cuts, (b) the intensity of the cut along the satellite footpoint for the black (optical) line only and (c) the energy and pictch angle range of particles with the top three representing the electrons and the bottom three the ions. In all particle spectrograms the satellite passes are from north to south (left to right). The equatorward edge of the auroral oval is visible in the all sky images for all four cases. This has been verified through examination of the time development of the aurora as well as that of the Reimei particle data over a longer period of time.

\section{Observations}

A representative all-sky image for each overpass is shown in Fig. 1. These show the range of diffuse auroral structures investigated, including the pulsating structures on 12 February 2007, and the very stationary east-west aligned dark structures on 1 March 2008. The dark line on each image indicates the path of the Reimei footpoint over the time interval investigated. The red square denotes the footpoint location at the time of the image, and the green square near the center of the image represents the magnetic zenith location. North is down and East is to the right. 

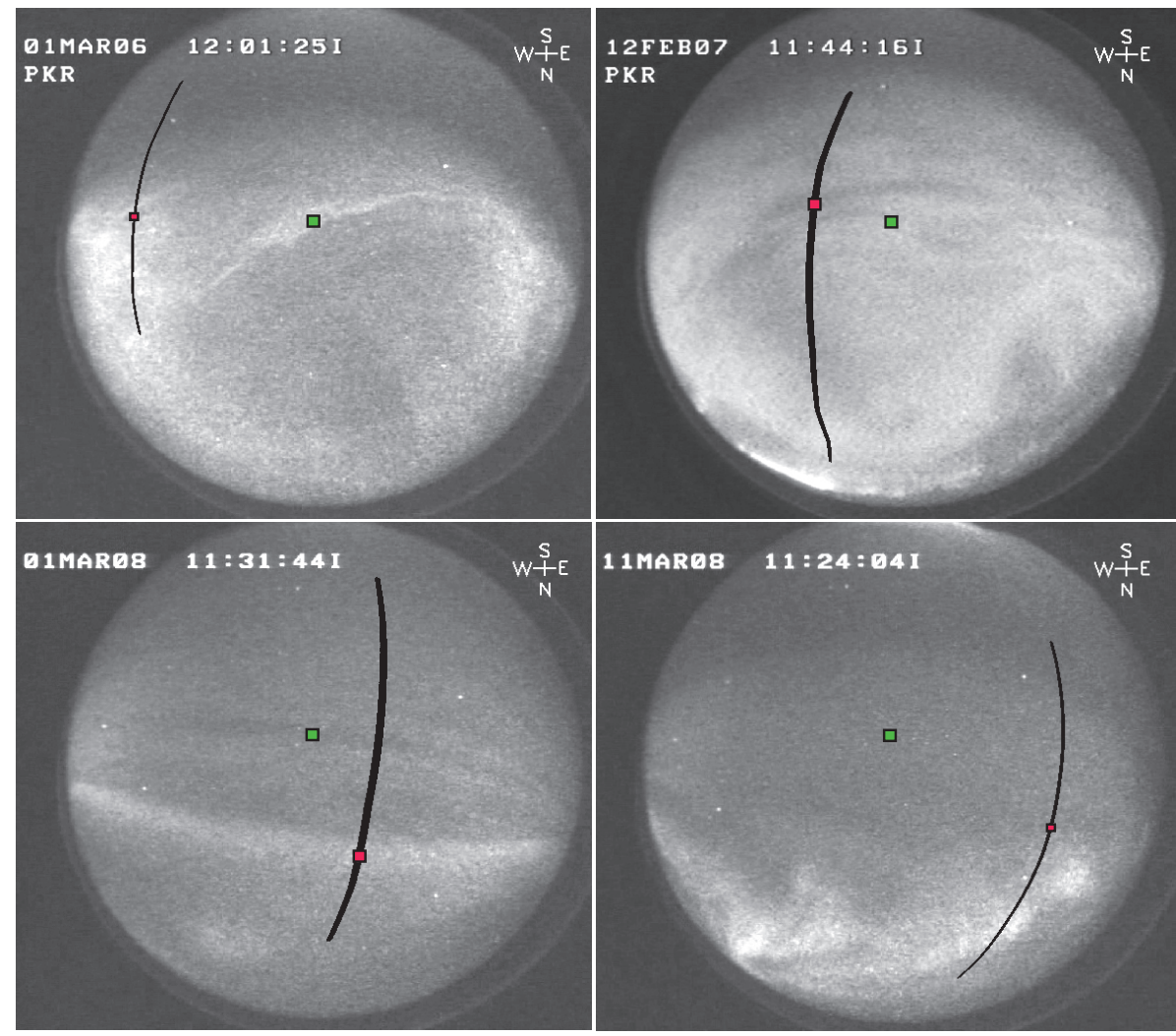

Fig. 1. Representative all-sky images for each overpass. Upper-left: 1 March 2006; upper-right: 12 February 2007; lower-left: 1 March 2008, and lower-right: 11 March 2008. The dark line on each image indicates the Reimei footpoint over the interval of interest, while the red square indicates the footpoint position at the time of the image. The green squares denote the position of the magnetic zenith. North is down and East is to the right.

Figure 2 shows the overview data for 1 March 2006 and 12 February 2007, illustrating the effectiveness of this type of keogram.

The 1 March 2006 overpass (left panel of Fig. 2) shows a sharp transition between bright diffuse aurora and dark sky with no significantly visible emissions. There is a faint arc structure that is spatially and temporally stationary. The sub-structure matches the fluxes of higher energy $(\sim 10 \mathrm{keV})$ electrons well. For example, near 12:01:25 the intensity of the aurora begins to drop rapidly, while the highest energy precipitating electrons drop out. Just seconds after, at 12:01:28, there is a thin bright structure that corresponds to a small increase in the high energy electron flux. Visible at the same time is a population of high energy $(>8 \mathrm{keV})$ ions, both precipitating and perpendicular, that drop out sharply at 12:01:30, where the characteristic energy of the electron population also begins to decrease sharply.

The 12 February 2007 overpass (right panel of Fig. 2), contains good examples of sub-structure which vary both spatially and temporally. This pass contains classic pulsating aurora, where each of the dark regions of the oscillations corresponds to a distinct dropout of the higher energy ( $\geq 5$ to $10 \mathrm{keV}$ ) portion of the precipitating electrons. "zoom1" denotes the time period to be examined in Fig. 4.

The 1 March 2008 overpass (left panel of Fig. 3) shows diffuse auroral structures (arcs) with no time variation. The bright arc corresponds to the region of $\sim 10 \mathrm{keV}$ precipitating electrons, and there are two distinct dropouts that correspond to the two dark regions traversed by the satellite. "zoom2" denotes the time period around one of those dark regions to be examined in Fig. 4.

Finally, the 11 March 2008 (right panel of Fig. 3) overpass is another example of the transition region at the equatorward edge of the aurora. The bright arc corresponds to the highest energy precipitating electron flux, the edge of which appears to be where the distinct boundary region is.

\section{Discussion}

Each case with a distinct dropout of the high energy precipitating electrons, corresponds to a dark region in the aurora at the satellite's magnetic footpoint. This is an indication that the luminous auroral features are caused by precipitation of 

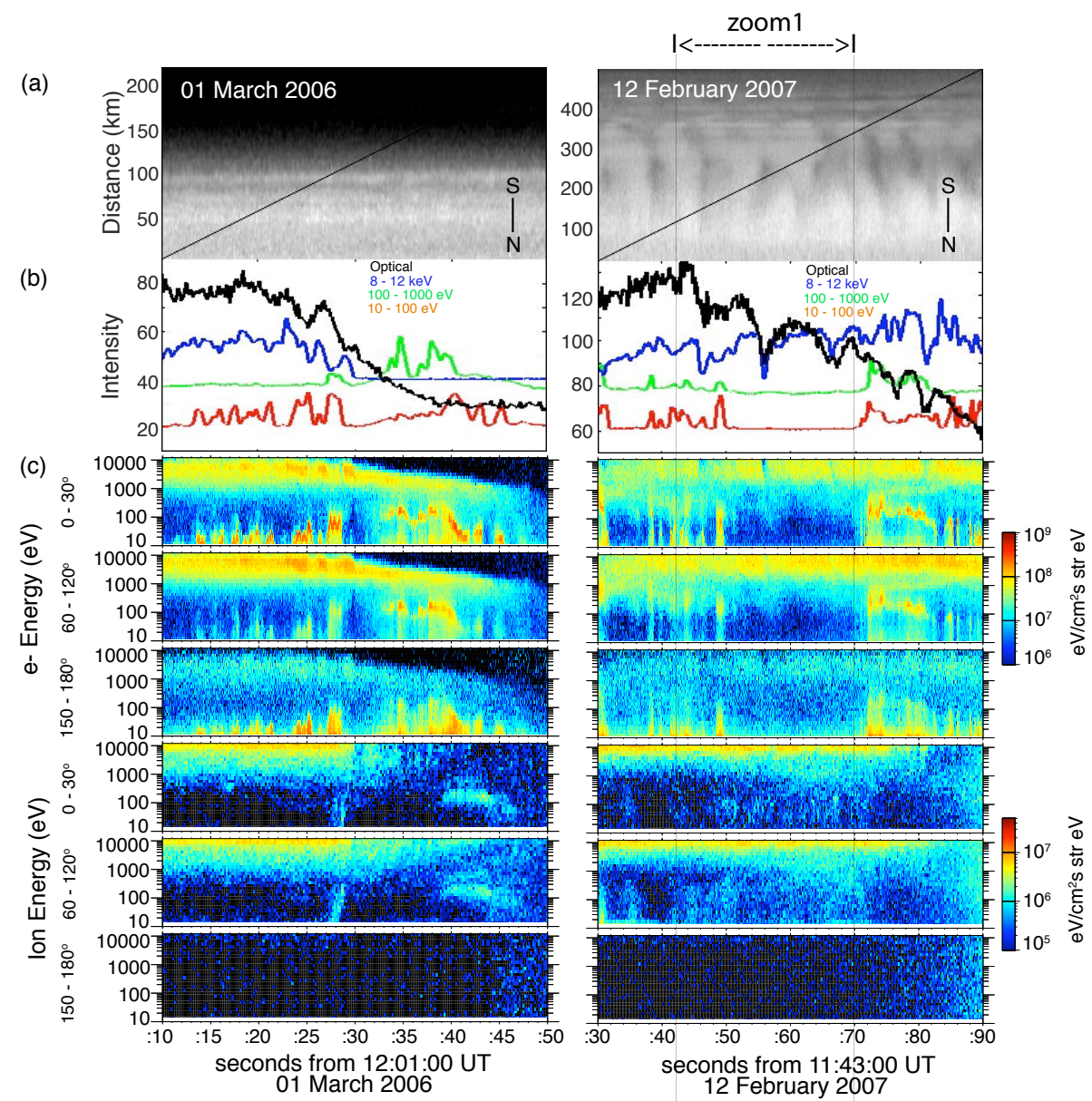

Fig. 2. Overview of data for 2 overpasses: Left column: 1 March 2006, Right column: 12 February 2007. (a) Keogram of auroral intensity along the Reimei footpoint (North at the bottom, South at the top). The distance along the satellite track, assuming $100 \mathrm{~km}$ altitude, is along the $y$-axis and time is along the $\mathrm{x}$-axis. The diagonal black line represents the location of the satellite footpoint at that time. (b) Black line: intensity at the footpoint location extracted from the optical signature. Three coloured lines: Summed flux of the downgoing electrons over the noted energy ranges, scaled for comparison. (c) Ion and electron data from the Reimei satellite. Zoom1 denotes one of the specific time periods shown in Fig. 4.

electrons with energy $\geq 8 \mathrm{keV}$. It should also be noted that the width of the dark structures is aspect angle sensitive, with an apparent width (contrast) that will decrease with increasing distance from magnetic zenith.

The 1 March 2006 overpass reveals a clear auroral boundary, likely the separation between large scale magnetospheric regions, possibly the equatorward edge of the plasmasheet. The broad in energy $(\leq 1 \mathrm{keV})$, counter-streaming electron populations that occur on both sides of this distinct boundary are likely Alfvén wave-accelerated (Chaston et al., 2008). There are qualitative differences between the two regions. On the plasmasheet side (12:01:10 to 12:01:30 UTC) they are bursty and localized in time and energy, while on the equatorward side (past 12:01:30 UTC) they are continuous and spread out in time and energy, filling in the background region below $1 \mathrm{keV}$.
The pulsating aurora case of 12 February 2007 also demonstrates boundaries. Near the poleward side of this interval, where the aurora is brighter and its variations are small, the low-energy electrons tend to be bursty and localized. Similarly, near the equatorward side, they become continuous and spread out. However, in the intervening region, there is a distinct lack of low energy electrons. Figure 2a shows that it corresponds to the largest and most distinct (largest difference in intensity between bright and dark regions) temporal variations. The high energy ions (downgoing $0^{\circ}$ to $30^{\circ}$ and perpendicular $60^{\circ}$ to $120^{\circ}$ ) are present until 11:44:20 UTC, which corresponds closely to the equatorward edge of the region with time-varying auroral structures. Moreover, there are still high energy precipitating electrons, as well as low energy electron populations in the region equatorward of the high energy ions (after 11:44:20 UTC), 


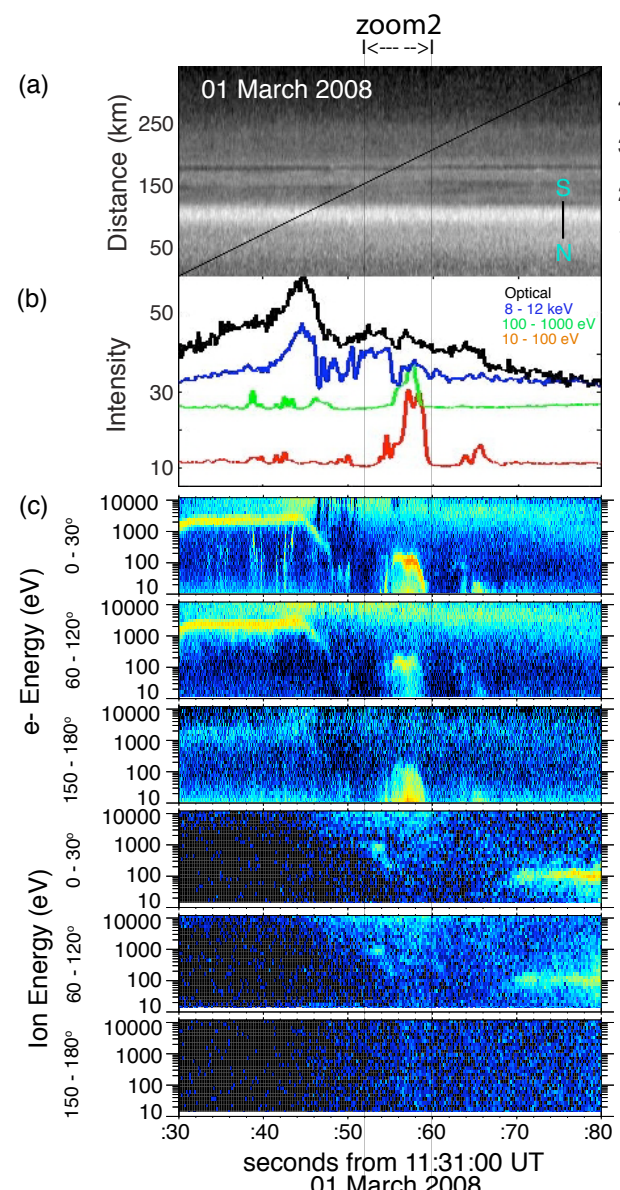

01 March 2008

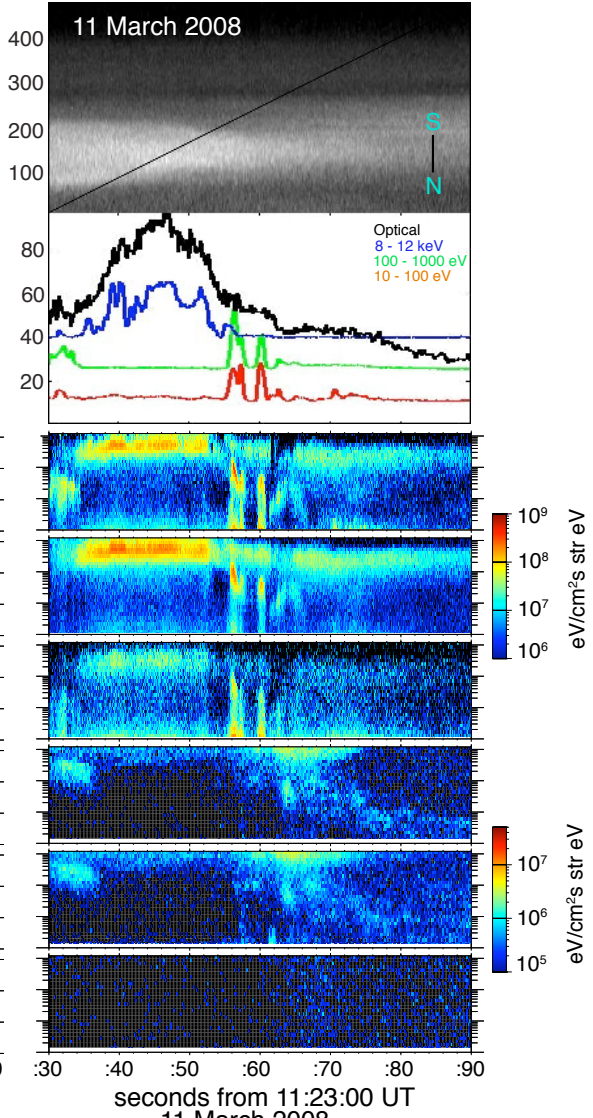

11 March 2008

Fig. 3. Same format as Fig. 2, with 1 March 2008 in the left panel and 11 March 2008 in the right panel. Zoom2 denotes one of the specific time periods shown in Fig. 4.

indicating that the electron plasmasheet extends earthward of the ions. The high energy electron distribution appears to have been cut off by the instrumental limit of $12 \mathrm{keV}$ and therefore it is possible that this event contains significant electron precipitation at energies greater than that, which are unobservable by Reimei.

There are two distinct lower energy electron populations on 1 March 2008 as well. The first is the mono-energetic $\sim 2 \mathrm{keV}$ population that exists in both the downgoing and perpendicular pitch angles, likely accelerated through a parallel potential drop. The other one from 11:31:54 to 11:32:00 UTC, extends all the way into the upgoing pitch angles. This population also displays characteristics of parallel potential acceleration, evidenced by the inverted- $\mathrm{V}$ shape in the precipitating pitch angles. The keogram in Fig. 3a again shows that this corresponds to the location of a distinct dark stripe in the aurora that remained stationary in space and time. This dark region corresponds to a drop out of the 8$12 \mathrm{keV}$ electrons. It is possible that there was enhanced wave activity in this dark region that led to the acceleration and isotropization of the low energy electron population. The meridian scanning photometer (MSP), also at Poker Flat, reveals that in the same region of the arc (assuming east-west alignment) there were enhanced $630.0 \mathrm{~nm}$ emissions, likely the result of these low energy precipitating electrons. Figure 5 shows the $630.0 \mathrm{~nm}$ MSP data for this time as well as the relative locations of the MSP scan and the satellite footpoint. The peak in the $630.0 \mathrm{~nm}$ emission occurs in the region just equatorward of the dark stripe, co-located with the mono-energetic $\sim 2 \mathrm{keV}$ downgoing electron population. Figure 3 (left column) shows what appears to be the low energy tail of downgoing and perpendicularly heated high energy ions in this region, with a peak energy greater than $12 \mathrm{keV}$. Figure 4 (right column) shows a high time resolution zoom in on this region. Also note that the dark region is associated with wave accelerated electrons, and significant fluxes of upgoing thermal electrons, both characteristics of auroral downward current regions (Marklund et al., 2001; Streltsov, 2007). However, a definitive identification cannot be made on the basis of these data. 

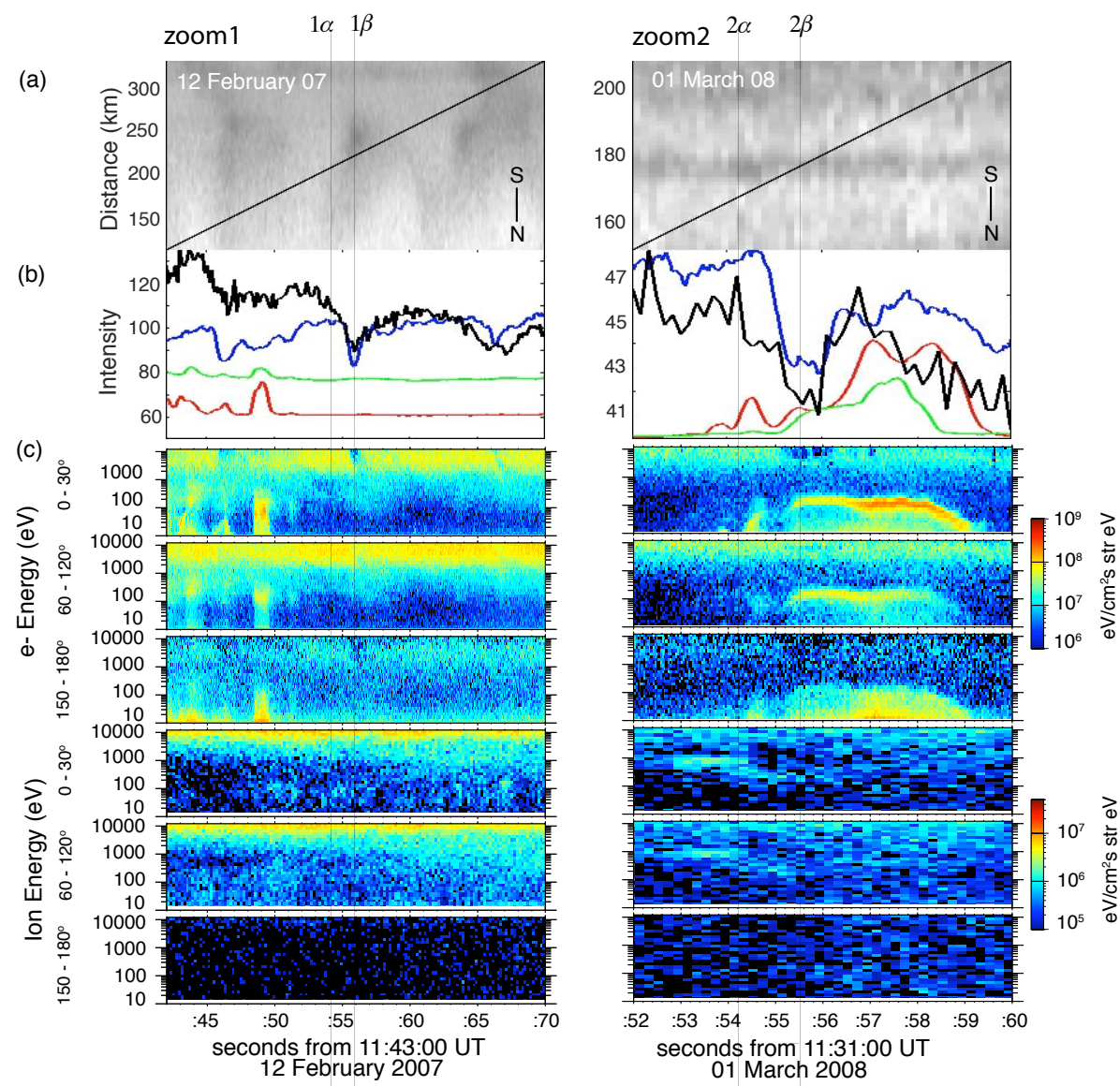

Fig. 4. Same format as Fig. 2, but showing two shorter time periods on 12 February 2007, starting at 11:43:42 UTC (left panel) and 1 March 2008, starting at 11:31:52 UTC (right panel). Four specific times are noted, $1 \alpha, 1 \beta, 2 \alpha$, and $2 \beta$, which are the times examined in more detail in Fig. 6.

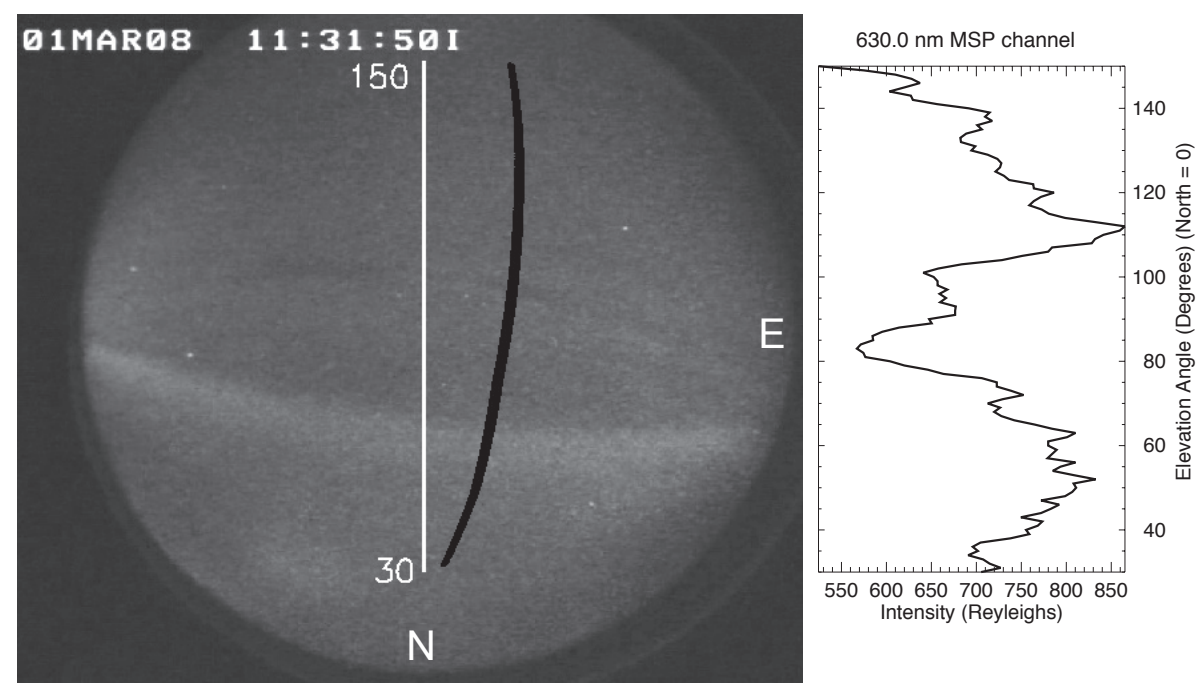

Fig. 5. All-sky image from 1 March 2008, showing the location of the MSP scan and the path of the Reimei footpoint. The line plot on the right shows the intensity of the $630.0 \mathrm{~nm}$ emissions, as a function of position along the image. 


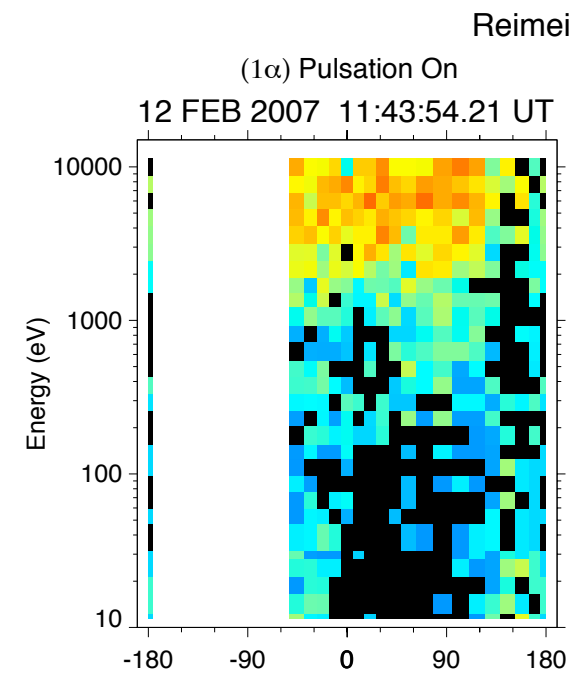

EISA (Electron)
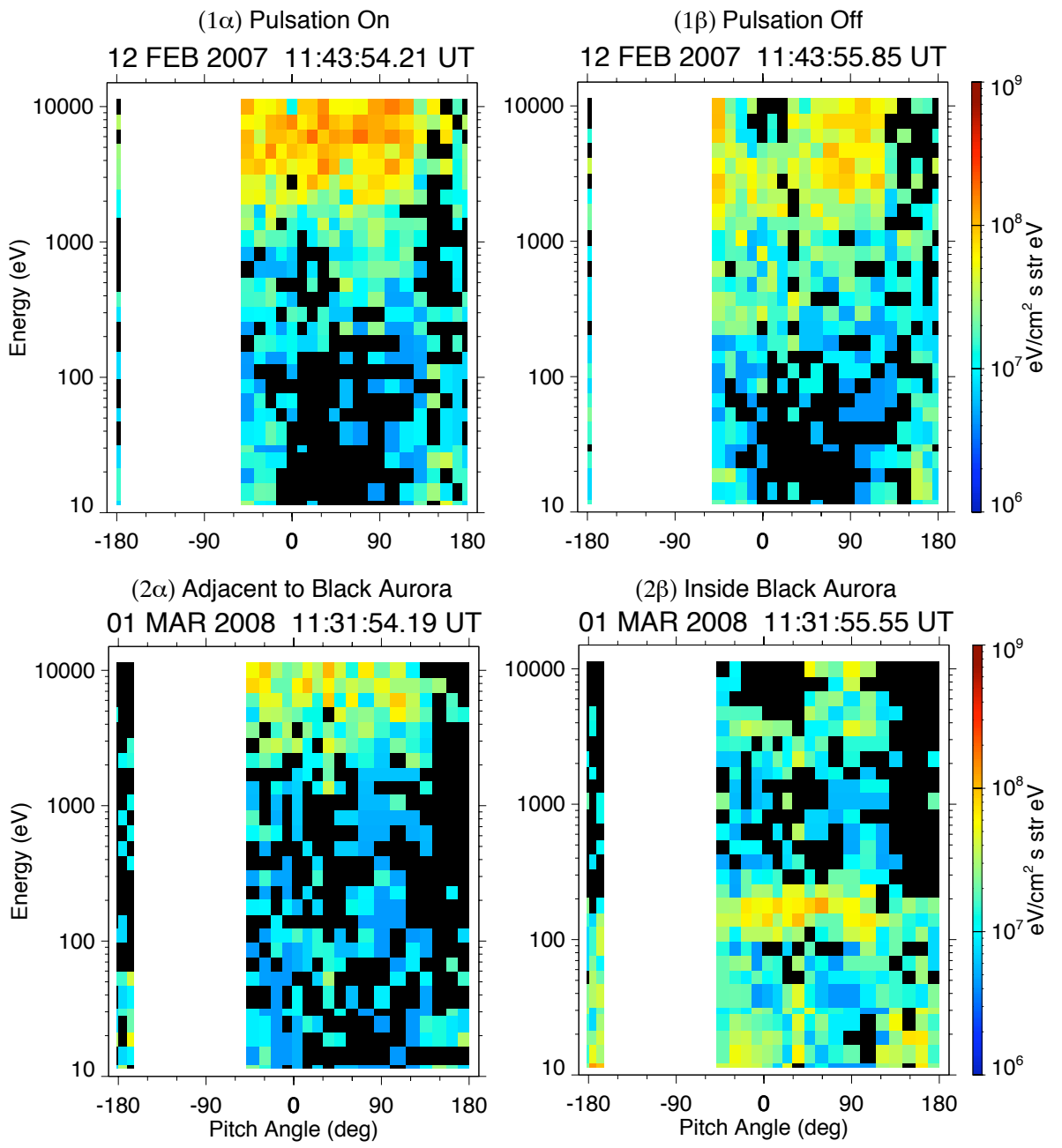

Fig. 6. Electron differential energy flux as a function of energy and pitch angle for a pulsating aurora case (1) and a stable black aurora case (2), both in the bright $(\alpha)$ and dark $(\beta)$ regions of the aurora.

11 March 2008, shows a bright arc corresponding to precipitating electrons with a characteristic energy of $\sim 6$ to $8 \mathrm{keV}$. There are two distinct low energy electron populations displaying characteristics of wave-acceleration (scattering). A distinct gap (11:24:02-11:24:05) in the high energy precipitating electrons, corresponds to a dark region in the aurora consisting of high energy $\sim 10 \mathrm{keV}$ precipitating and perpendicular ions.

Figure 4 examines "zoom1" from Fig. 2 (12 February 2007) and "zoom2" from Fig. 3 (1 March 2008). They illustrate a time varying structure, pulsating aurora, and the smallscale features of a distinct stationary black auroral structure, respectively. There is a close match between the high energy electrons and the auroral structures, demonstrated by the three coloured lines in panel (b). The energy range of 8 to $12 \mathrm{keV}$ demonstrates the best correlation with the optical data. Energy ranges other than the ones shown were exam- ined, such as the 5 to $8 \mathrm{keV}$ electrons which correlated to the optical data for some events, as opposed to the 8 to $12 \mathrm{keV}$ electrons that showed correlations in all four events.

The pulsating aurora example, contained dropouts of electron precipitation that extended down to $\sim 2 \mathrm{keV}$, consistent with the the black aurora observations of Peticolas et al. (2002). It has been suggested that black aurora is caused by a localized suppression of chorus wave scattering, since lower and upper band chorus waves are effective at scattering higher energy ( $\geq 2-4 \mathrm{keV}$ ) electrons, while electron cyclotron harmonic $(\mathrm{ECH})$ waves can only scatter lower energy ( $\leq 2-4 \mathrm{keV})$ electrons (Peticolas et al., 2002; Sergienko et al., 2008; Su et al., 2009). However, there is increasing evidence that pulsating aurora does not originate in the equatorial plane. Watanabe et al. (2007) examined conjugate data from Iceland and Antarctica and concluded that the conjugacy of pulsating aurora is generally poor. Sato et al. (2004), 
using FAST data and conjugate ground-based imaging, concluded that the generation of the modulations occurred far Earthward of the magnetospheric equatorial plane. This suggests that magnetosphere-ionosphere coupling processes are important in generating the pulsating aurora.

Recently, Ni et al. (2008) examined the electron scattering rates for lower and upper band chorus, using the formulation of Glauert and Horne (2005). They found that upper band chorus is most effective at scattering electrons with energies less than $\sim 5 \mathrm{keV}$, while lower band chorus is effective at scattering higher energy ( $\geq 5 \mathrm{keV}$ ) electrons. This is consistent with the diffuse auroral observations presented with this work. The dark regions of the aurora correspond to drop outs of the higher energy ( $\geq 8 \mathrm{keV}$ ) electrons, suggesting that lower band chorus pitch angle scattering is responsible for the majority of the visible diffuse aurora, while the darker regions are consistent with localized suppressions of lower band chorus, but not upper band chorus or ECH waves.

To examine the possible existence of partial double loss cones in both the spatial and temporal dark structures, after Peticolas et al. (2002), two specific times where noted for each event shown in Fig. 4. (1) refers to the pulsating aurora and (2) to the black aurora, while the bright areas are denoted by $(\alpha)$ and the dark regions by $(\beta)$. These times are examined in Fig. 6, where the electron differential energy flux as a function of energy and pitch angle is presented for each case. For the 12 February 2007 event, $1 \alpha$ denotes a time when the satellite is in the bright region of a pulsation, while $1 \beta$ when the satellite is in the dark region between the pulsating. The 1 March 2008 event, shows a stationary black auroral structure, where $2 \alpha$ is a time in the luminous diffuse aurora adjacent to the black stripe, and $2 \beta$ inside the black structure. Figure 6 verifies that there indeed exist partial double loss cones for the dark structures in both pulsating and black aurora. The main difference between bright and dark regions is in the downgoing electrons with energies greater than $5 \mathrm{keV}$. The implication of this observation is that the processes causing the lack of precipitation in the black aurora are only acting on the downgoing, $\geq 5 \mathrm{keV}$, electron population.

\section{Conclusions}

In cases with non time-varying auroral structures (over $\sim 1$ min time scales) the low energy electrons (broad in both energy and pitch angle) appear to be co-located with the dark regions of the aurora that are also associated with the dropouts of the high energy electrons. In the example with time-varying auroral structures (12 February 2007), there appear to be no low energy electrons in the region with the most intense temporal variations (largest intensity variations). Figures 2 and 3 show a clear transition between two different plasma populations, one with high energy electrons and ions, and one with no ions and large fluxes of low energy electrons.
These four overpasses illustrate the abundance of smallscale structure within the diffuse aurora and the distinct differences in the plasma associated with each region. In all cases, the dark auroral features are coincident with dropouts of high energy electrons verifying that these aurorae are indeed associated with $\geq 8 \mathrm{keV}$ electrons that do not exhibit inverted-V characteristics. Therefore, they are not accelerated through a parallel potential drop but instead scattered into the loss cone from trapped magnetospheric particles, identifying the associated aurora as diffuse. In addition, examination of the electron distributions as a function of energy and pitch angle reveal partial double loss-cones, indicating that the processes generating the observed black aurorae only act in the downgoing, $\geq 5 \mathrm{keV}$, electrons.

These observations show, in unprecedented highresolution detail, specific diffuse auroral structures and their corresponding electron and ion distributions. Continued observations with both in situ plasma measurements and conjugate ground-based auroral imaging will provide further insight into the electron distributions that are responsible for a wider range of observable auroral features.

Acknowledgements. The authors would like to thank the entire Reimei satellite team in Japan for their prompt assistance in coordinating observations. This work was supported in part by an internal research grant at Southwest Research Institute and National Science Foundation grant \# ATM-0836410.

Topical Editor K. Kauristie thanks B. Gustavsson and another anonymous referee for their help in evaluating this paper.

\section{References}

Asamura, K., Tsujita, D., Tanaka, H., Saito, Y., Mukai, T., and Hirahara, M.: Auroral particle instrument onboard the index satellite, Adv. Space Res., 32, 375-378, doi:10.1016/S0273-1177(03) 90275-4, 2003.

Blixt, E. M. and Kosch, M. J.: Coordinated optical and EISCAT observations of black aurora, Geophys. Res. Lett., 31, 6813, doi: 10.1029/2003GL019244, 2004.

Blixt, E. M., Kosch, M. J., and Semeter, J.: Relative drift between black aurora and the ionospheric plasma, Ann. Geophys., 23, 1611-1621, 2005, http://www.ann-geophys.net/23/1611/2005/.

Chaston, C. C., Salem, C., Bonnell, J. W., Carlson, C. W., Ergun, R. E., Strangeway, R. J., and McFadden, J. P.: The Turbulent Alfvénic Aurora, Phys. Rev. Lett., 100, 175003, doi: 10.1103/PhysRevLett.100.175003, 2008.

Dashkevich, Zh. V., Ivanov, V. E., and Khudukon, B. Z.: Features of stable diffuse arcs observed by means of auroral tomography, Ann. Geophys., 25, 1131-1139, 2007, http://www.ann-geophys.net/25/1131/2007/.

Glauert, S. A. and Horne, R. B.: Calculation of pitch angle and energy diffusion coefficients with the PADIE code, J. Geophys. Res., 110, 4206, doi:10.1029/2004JA010851, 2005.

Haerendel, G., Frey, H. U., Bauer, O. H., Rieger, E., Clemmons, J., Boehm, M. H., Wallis, D. D., and Luhr, H.: Inverted-V events 
simultaneously observed with the Freja satellite and from the ground, Geophys. Res. Lett., 21, 1891-1894, 1994.

Hallinan, T. J., Kimball, J., Stenbaek-Nielsen, H. C., Lynch, K., Arnoldy, R., Bonnell, J., and Kintner, P.: Relation between optical emissions, particles, electric fields, and Alfvén waves in a multiple rayed arc, J. Geophys. Res., 106, 15445-15454, 2001.

Horne, R. B. and Thorne, R. M.: Electron pitch angle diffusion by electrostatic electron cyclotron harmonic waves: The origin of pancake distributions, J. Geophys. Res., 105, 5391-5402, doi: 10.1029/1999JA900447, 2000.

Horne, R. B., Thorne, R. M., Meredith, N. P., and Anderson, R. R.: Diffuse auroral electron scattering by electron cyclotron harmonic and whistler mode waves during an isolated substorm, J. Geophys. Res., 108, 1290, doi:10.1029/2002JA009736, 2003.

Johnstone, A. D., Walton, D. M., Liu, R., and Hardy, D. A.: Pitch angle diffusion of low-energy electrons by whistler mode waves, J. Geophys. Res., 98, 5959-5967, 1993.

Kimball, J. and Hallinan, T. J.: Observations of black auroral patches and of their relationship to other types of aurora, J. Geophys. Res., 103, 14671-14682, 1998.

Maggs, J. E. and Davis, T. N.: Measurements of the thicknesses of auroral structures, Planet. Space Sci., 16, 205-209, 1968.

Marklund, G. T., Ivchenko, N., Karlsson, T., Fazakerley, A., Dunlop, M., Lindqvist, P.-A., Buchert, S., Owen, C., Taylor, M., Vaivalds, A., Carter, P., André, M., and Balogh, A.: Temporal evolution of the electric field accelerating electrons away from the auroral ionosphere, Nature, 414, 724-727, 2001.

Meredith, N. P., Horne, R. B., Johnstone, A. D., and Anderson, R. R.: The temporal evolution of electron distributions and associated wave activity following substorm injections in the inner magnetosphere, J. Geophys. Res., 105, 12907-12918, doi: 10.1029/2000JA900010, 2000.

Meredith, N. P., Horne, R. B., Thorne, R. M., and Anderson, R. R.: Survey of upper band chorus and ECH waves: Implications for the diffuse aurora, J. Geophys. Res., 114, 7218, doi: 10.1029/2009JA014230, 2009.

Ni, B., Thorne, R. M., Shprits, Y. Y., and Bortnik, J.: Resonant scattering of plasma sheet electrons by whistler-mode chorus: Contribution to diffuse auroral precipitation, Geophys. Res. Lett., 35, 11106, doi:10.1029/2008GL034032, 2008.
Peticolas, L. M., Hallinan, T. J., Stenbaek-Nielsen, H. C., Bonnell, J. W., and Carlson, C. W.: A study of black aurora from aircraftbased optical observations and plasma measurements on FAST, J. Geophys. Res., 107, 1217, doi:10.1029/2001JA900157, 2002.

Royrvik, O.: Pulsating aurora: Local and global morphology, PhD thesis, Alaska Univ., College., 1976.

Sato, N., Wright, D. M., Carlson, C. W., Ebihara, Y., Sato, M., Saemundsson, T., Milan, S. E., and Lester, M.: Generation region of pulsating aurora obtained simultaneously by the FAST satellite and a Syowa-Iceland conjugate pair of observatories, J. Geophys. Res. (Space Physics), 109, 10201, doi:10.1029/2004JA010419, 2004.

Schoute-Vanneck, H., Scourfield, M. W. J., and Nielsen, E.: Drifting black aurorae?, J. Geophys. Res., 95, 241-246, doi:10.1029/ JA095iA01p00241, 1990.

Sergienko, T., Sandahl, I., Gustavsson, B., Andersson, L., Brändstrm, U., and Steen, Å.: A study of fine structure of diffuse aurora with ALIS-FAST measurements, Ann. Geophys., 26, 3185-3195, 2008, http://www.ann-geophys.net/26/3185/2008/.

Stenbaek-Nielsen, H. C., Hallinan, T. J., Osborne, D. L., Kimball, J., Chaston, C., McFadden, J., Delory, G., Temerin, M., and Carlson, C. W.: Aircraft observations conjugate to FAST: Auroral arc thicknesses, Geophys. Res. Lett., 25, 2073-2076, 1998.

Stenbaek-Nielsen, H. C., Hallinan, T. J., and Peticolas, L.: Why do auroras look the way they do?, EOS Transactions, 80, 193-193, doi:10.1029/99EO00138, 1999.

Streltsov, A. V.: Narrowing of the discrete auroral arc by the ionosphere, J. Geophys. Res., 112, 10218, doi:10.1029/ 2007JA012402, 2007.

Su, Z., Zheng, H., and Wang, S.: Evolution of electron pitch angle distribution due to interactions with whistler mode chorus following substorm injections, J. Geophys. Res., 114, 8202, doi: 10.1029/2009JA014269, 2009.

Trondsen, T. S. and Cogger, L. L.: High-resolution television observations of black aurora, J. Geophys. Res., 102, 363-378, 1997.

Watanabe, M., Kadokura, A., Sato, N., and Saemundsson, T.: Absence of geomagnetic conjugacy in pulsating auroras, Geophys. Res. Lett., 34, 15107, doi:10.1029/2007GL030469, 2007. 